\title{
Weighted Nash inequalities
}

\section{Dominique Bakry, François Bolley, Ivan Gentil and Patrick Maheux}

\begin{abstract}
Nash or Sobolev inequalities are known to be equivalent to ultracontractive properties of Markov semigroups, hence to uniform bounds on their kernel densities. In this paper, following work of F.-Y. Wang, we present a simple and extremely general method, based on weighted Nash inequalities, for obtaining non-uniform bounds on kernel densities. Such bounds imply control of the trace or the Hilbert-Schmidt norm of the heat kernels. We illustrate the method on the heat kernel on $\mathbb{R}$ naturally associated with the measure with density $C_{a} \exp \left(-|x|^{a}\right)$, with $1<a<2$, for which uniform bounds are known not to hold.
\end{abstract}

\section{Introduction}

The classical Nash inequality in $\mathbb{R}^{n}$ can be stated as

$$
\|f\|_{2}^{1+n / 2} \leq C_{n}\|f\|_{1}\|\nabla f\|_{2}^{n / 2}
$$

for all smooth functions $f$ (with compact support for instance) where the norms are computed with respect to the Lebesgue measure. This inequality was introduced by J. Nash in 1958 (see [24]) to obtain regularity properties of the solutions to parabolic partial differential equations. The optimal constant $C_{n}$ was computed more recently in [13].

In the more general setting of a symmetric Markov semigroup $\left(P_{t}\right)_{t \geq 0}$ one has to replace $\|\nabla f\|_{2}^{2}$ by the Dirichlet form $\mathcal{E}(f, f)$ associated with its generator. Inequality (1.1) implies smoothing properties of the Markov semigroup in the following way: given a function $f$, then $\varphi(t)=\left\|P_{t} f\right\|_{2}^{2}$ has derivative $\varphi^{\prime}(t)=-2 \mathcal{E}\left(P_{t} f, P_{t} f\right)$, so, by the Nash inequality (1.1),

$$
\varphi(t)^{1+n / 2} \leq C_{n}^{2}\left\|P_{t} f\right\|_{1}^{2}\left(-\varphi^{\prime}(t) / 2\right)^{n / 2} \leq C_{n}^{2}\|f\|_{1}^{2}\left(-\varphi^{\prime}(t) / 2\right)^{n / 2}
$$

Integrating leads first to the bound $\left\|P_{t} f\right\|_{2} \leq C^{\prime} t^{-n / 4}\|f\|_{1}$ for $t>0$, and then to $\left\|P_{t} f\right\|_{\infty} \leq C^{\prime} t^{-n / 4}\|f\|_{2}$, by duality and symmetry of the semigroup. This finally

Mathematics Subject Classification (2010): 35P05; 47D07; 35P15; 60J60.

Keywords: Nash inequality, super-Poincaré inequality, heat kernel, ultracontractivity. 
implies the classical uniform bound

$$
\left\|P_{t} f\right\|_{\infty} \leq C^{\prime 2} t^{-n / 2}\|f\|_{1}
$$

for $t>0$ by semigroup properties. In turn this implies uniform bounds on the kernel density of the semigroup such as

$$
\left|p_{t}(x, y)\right| \leq C^{\prime 2} t^{-n / 2}
$$

for all $x, y$ and $t>0$.

Depending on whether the reference measure is finite or not, Nash inequalities take the general form

$$
\|f\|_{2}^{1+n / 2} \leq\|f\|_{1}\left[a \mathcal{E}(f, f)+b\|f\|_{2}^{2}\right]^{n / 4}
$$

where $n$ no longer needs to be an integer. These are some of the many forms of the celebrated Sobolev inequality

$$
\|f\|_{2 n /(n-2)} \leq a \mathcal{E}(f, f)+b\|f\|_{2}^{2}
$$

for $n>2$, see [6], [25]. Up to constants, these inequalities are all equivalent to the ultracontractive bound

$$
\left\|P_{t} f\right\|_{\infty} \leq C t^{-n / 2}\|f\|_{1}, \quad 0<t \leq 1
$$

on the Markov semigroup associated to the Dirichlet form $\mathcal{E}$, hence to uniform bounds on the kernel density of the semigroup $P_{t}$ with respect to the reference measure. See [9], [12], [14], [15] and [26] among many articles on this topic.

The Nash inequalities (1.4) do not give the optimal constant $C$ in (1.6). The optimal contractive bounds $\left\|P_{t} f\right\|_{q} \leq C_{p, q, n}(t)\|f\|_{p}$ for the classical heat equation in $\mathbb{R}^{n}$ can be obtained from the Euclidean logarithmic Sobolev inequality (see [2], [21]), but the Nash inequality is the easiest and the most intuitive way to get ultracontractive bounds such as (1.6).

The inequalities (1.4) have been studied by F.-Y. Wang in [27] as part of a more general family of inequalities, called super-Poincaré inequalities, of the form

$$
\|f\|_{2}^{2} \leq a \mathcal{E}(f, f)+b(a)\|f\|_{1}^{2}
$$

for $a>a_{0}$, where $b$ is a non-negative function. Optimising in (1.7) over the parameter $a$ leads to

$$
\frac{\|f\|_{2}^{2}}{\|f\|_{1}^{2}} \leq \psi\left(\frac{\mathcal{E}(f, f)}{\|f\|_{1}^{2}}\right)
$$

where $\psi(x)=\inf _{a}\{a x+b(a)\}$ is an increasing concave function, or equivalently

$$
\phi\left(\frac{\|f\|_{2}^{2}}{\|f\|_{1}^{2}}\right) \leq \frac{\mathcal{E}(f, f)}{\|f\|_{1}^{2}}
$$


for an increasing convex function $\phi$. Following the argument leading to (1.2), this implies the ultracontractive bound

$$
\left\|P_{t} f\right\|_{\infty} \leq U^{-1}(t)\|f\|_{1},
$$

for all $t>0$, where $U(t)=\int_{t}^{\infty} 1 / \phi(x) d x$ is well defined under adequate assumptions on $\phi$ (see [14]).

The generalized Nash inequalities (1.7) are also a powerful tool to obtain spectral properties of the generator defining the Dirichlet form (see [27]); in particular they imply that its essential spectrum is empty. When the reference measure has finite mass, they also provide additional information about fields of concentration, asymptotic behavior and isoperimetry of the measure, as in [8]. They belong to a large family of functional inequalities such as the logarithmic Sobolev and the Poincaré inequalities, and have been studied in many recent papers such as [22], [30].

This article is devoted to a more general situation in which the semigroup is not ultracontractive, so that one cannot expect uniform bounds on its kernel density, as in (1.3). For instance, the Ornstein-Uhlenbeck semigroup on $\mathbb{R}^{n}$ (which is probably the most studied semigroup on $\mathbb{R}^{n}$, besides the classical heat semigroup) is not ultracontractive; in fact, according to a famous result of E. Nelson, it is only hypercontractive (see [1], for example). Observe that, according to the celebrated theorem of L. Gross [17], the corresponding hypercontractive bounds are equivalent to a logarithmic Sobolev inequality for the Gaussian measure (which is weaker than the Sobolev inequality (1.5)). Of course the Ornstein-Uhlenbeck kernel is explicit, so it is useless to estimate it, but, for many other examples, pointwise estimates on the kernels are an interesting and not so easy issue. There is a very large literature on this problem, see [15] and the references therein.

Non-uniform estimates on the density of the heat kernel can provide useful information on the semigroup. For example, let us consider a symmetric semigroup $\left(P_{t}\right)_{t \geq 0}$ which can be represented by a density $p_{t}(x, y)$ with respect to an invariant measure $\mu$, that is, such that

$$
P_{t} f(x)=\int_{E} f(y) p_{t}(x, y) d \mu(y)
$$

for all $x$ and $t>0$. Then the operator $P_{t}$ is in the trace class and therefore has a discrete spectrum as soon as $p_{t}(x, x) \in \mathcal{L}^{1}(\mu)$; moreover, estimates on the spectrum can be obtained as detailed below.

In the general situation when the kernel density might not be uniformly bounded, the classical Nash inequality (1.1) is not adapted, and the main idea in this article is to use the generalized Nash inequality (1.8), modified with a weight depending on the expected estimate. Depending on the generator of the heat kernel and the reference measure considered in the $\mathcal{L}^{p}$ norms, we shall look for a positive function $V$ and an increasing and convex function $\phi$ such that

$$
\phi\left(\frac{\|f\|_{2}^{2}}{\|f V\|_{1}^{2}}\right) \leq \frac{\mathcal{E}(f, f)}{\|f V\|_{1}^{2}}
$$

for all $f$. Such an inequality will be called a weighted Nash inequality. 
We shall look for weight functions $V$ satisfying the subharmonic condition $L V \leq c V$ where $L$ is the infinitesimal generator of the semigroup; this assumption is very close to (but easier to satisfy) the condition on Lyapunov functions recently used by the first author, F. Barthe, P. Cattiaux and A. Guillin in [3] and [5] to prove functional inequalities such as the Poincaré and super-Poincaré inequalities. Here is a key difference between our approach and theirs: the Lyapunov functions used in the present work appear explicitly in the functional inequalities themselves, whereas in the works mentioned above they are only a tool to get the sought after functional inequalities, but they do not explicitly appear in the final estimates; they are used like a catalyst to derive them. We will prove that the weighted Nash inequality (1.10) and the subharmonic condition on the weight function $V$ imply the non-uniform estimate

$$
p_{t}(x, y) \leq K(t, \phi, c) V(x) V(y)
$$

of the heat kernel, for a positive function $K$.

After completing this work, we learned from F.-Y. Wang that he made a similar study under weighted super-Poincaré inequalities, in the framework of "intrinsic ultracontractivity": in particular our Theorem 3.5 and the converse Theorem 3.9 are strongly related to Theorem 3.3 in [28] (see also [31]). However, to have a complete and simple picture, we shall state and prove them in our context.

\section{Framework and outline of the work}

This work is devoted to properties of symmetric Markov semigroups $\left(P_{t}\right)_{t \geq 0}$. On a given measure space $(E, \mathcal{E}, \mu)$, a symmetric Markov semigroup is a family of positivity preserving operators acting on bounded measurable functions, which preserve constant functions, and are moreover symmetric in $\mathcal{L}^{2}(\mu)$. In the main application of Section 5 , the measure $\mu$ will be a probability measure, but it may also be a measure with infinite mass. The operators $P_{t}$ are contractions in $\mathcal{L}^{1}(\mu)$ and $\mathcal{L}^{\infty}(\mu)$, so are contractions in any $\mathcal{L}^{p}(\mu)$ with $1 \leq p \leq \infty$. The semigroup property consists in the identity $P_{t} \circ P_{s}=P_{t+s}$ for any $s$ and $t$ in $\mathbb{R}_{+}$, together with a continuity assumption at $t=0$, for example here that for any $f \in \mathcal{L}^{2}(\mu), P_{t} f$ converges to $f$ in $\mathcal{L}^{2}(\mu)$ when $t$ converges to 0 . We shall assume that, for all $t, P_{t}$ has a kernel, which is the case when $E$ is a Polish space.

Symmetric Markov semigroups appear naturally as the laws of Markov processes $\left(X_{t}\right)_{t>0}$ on $E$ which are reversible in time: for example, in the case when $\mu$ is a probability measure, this means that for any $T>0$, the law of the process $\left(X_{t}, 0 \leq t \leq T\right)$, where the law of $X_{0}$ is $\mu$, is the same as the law of the process $\left(X_{T-t}, 0 \leq t \leq T\right)$.

They also appear naturally when solving a heat equation

$$
\partial_{t} u=L u, \quad u(x, 0)=f(x)
$$

on $E \times[0, \infty)$; here $L$ is a (unbounded) self-adjoint operator satisfying the maximum principle and $L 1=0$, for example a second order differential sub-elliptic operator 
with no 0 -order term on an open set on $\mathbb{R}^{n}$ or a manifold; in this case, and under mild hypotheses, the solution may be represented as

$$
u(x, t)=P_{t} f(x) .
$$

By the Hille-Yosida theory, the operator $P_{t}$ has a derivative $L$ at $t=0$ which is defined in a domain dense in $\mathcal{L}^{2}(\mu)$. Moreover $P_{t}=\exp (t L)$ and $L$ is self-adjoint since $P_{t}$ is symmetric, see [32] for instance. Also $P_{t}$ is a contraction in $\mathcal{L}^{2}(\mu)$, so that the spectrum of $L$ lies in $(-\infty, 0]$.

Under our assumptions, for all $t>0$ the operator $P_{t}$ is represented by a kernel density $p_{t}(x, y)$ with respect to the reference measure $\mu$, in the sense that there exists a non-negative symmetric function $p_{t}$ on $E \times E$ such that

$$
P_{t} f(x)=\int_{E} f(y) p_{t}(x, y) d \mu(y)
$$

for $\mu$ almost every $x$ in $E$. Then the semigroup property $P_{t} \circ P_{s}=P_{t+s}$ may be translated into the celebrated Chapman-Kolmogorov equation

$$
\int_{E} p_{t}(x, y) p_{s}(y, z) d \mu(y)=p_{t+s}(x, z)
$$

for $\mu \otimes \mu$ almost every $(x, z)$ in $E \times E$.

Moreover, as soon as the kernel density $p_{t}(x, y)$ is in $\mathcal{L}^{2}(\mu \otimes \mu)$, the operator $P_{t}$ is Hilbert-Schmidt on $\mathcal{L}^{2}(\mu)$ (see [19] for instance). In particular $P_{t}$ has a discrete spectrum $\left(\mu_{n}(t)\right)_{n \in \mathbb{N}}$ associated to a sequence of orthonormal eigenfunctions $\left(e_{n}\right)_{n \in \mathbb{N}}$ in $\mathcal{L}^{2}(\mu)$. In this case,

$$
p_{t}(x, y)=\sum_{n} \mu_{n}(t) e_{n}(x) e_{n}(y)
$$

and the series converges since

$$
\sum_{n} \mu_{n}(t)^{2}=\int_{E \times E} p_{t}(x, y)^{2} d \mu(x) d \mu(y)<+\infty .
$$

Moreover,

$$
\int_{E \times E} p_{t}(x, y)^{2} d \mu(x) d \mu(y)=\int_{E} p_{2 t}(x, x) d \mu(x)
$$

so that $P_{2 t}$ is in the trace class. Of course such estimates can be established only for $t>0$.

Since $P_{t}=\exp (t L)$, this just shows that $L$ itself has a discrete spectrum $\left(-\lambda_{n}\right)_{n \in \mathbb{N}}$, with $\lambda_{n} \geq 0$ and $\lambda_{0}=0$, such that $\mu_{n}(t)=e^{-\lambda_{n} t}$. We see from the estimate $(2.1)$ how control of $p_{t}(x, x)$ or $p_{t}(x, y)$ may lead to control of the spectrum $\left(\mu_{n}(t)\right)_{n \in \mathbb{N}}$ of $P_{t}$, hence of the spectrum $\left(\lambda_{n}\right)_{n \in \mathbb{N}}$ of $L$.

In general, as explained above, it is not easy to show the existence of the density $p_{t}(x, y)$ or to obtain such control on it. The classical situation in which $P_{t}$ 
is Hilbert-Schmidt is when $\mu$ has finite mass and $p_{t}$ is bounded. For example, under the Nash inequality (1.1) or (1.8), then according to the ultracontractive bound (1.9) the operator $P_{t}$ is bounded from $\mathcal{L}^{1}(\mu)$ into $\mathcal{L}^{\infty}(\mu)$ with norm $C_{t}$. In this case $P_{t}$ may be represented by a kernel density $p_{t}$ which is $\mu \otimes \mu$ almost surely bounded by the same constant $C_{t}$ under a mild assumption on $(E, \mathcal{E}, \mu)$ (for instance if $\mathcal{E}$ is generated by a countable family, up to zero measure sets, see Lemma 4.3 in [2]). Spaces $(E, \mathcal{E}, \mu)$ for which this holds will be called nice measure spaces. They include Polish spaces on which Markov semigroups can be represented by a kernel.

This work is devoted to the case of non-ultracontractive semigroups, that is, of not necessarily bounded kernel densities. We shall replace the Nash inequality by the weighted Nash inequality (1.10) with a weight $V$ such that $L V \leq c V$ to obtain the existence of a density $p_{t}$ which satisfies

$$
p_{t}(x, y) \leq K(t, \phi, c) V(x) V(y) .
$$

See Proposition 3.1, Theorem 3.5 and Corollary 3.7.

In Section 4 we give a simple illustration of this method, see Theorem 4.1. There we deduce the following universal bound on $\mathbb{R}^{n}$ from the classical Nash inequality (1.1): if the invariant measure $\mu$, not necessarily finite, has a positive density $\rho$, then

$$
\|f\|_{2}^{2+\frac{4}{n}} \leq C_{n}\|f V\|_{1}^{\frac{4}{n}}\left(\mathcal{E}(f, f)+\int_{\mathbb{R}^{n}} \frac{L V}{V} f^{2} d \mu\right),
$$

where $V=\rho^{-1 / 2}$. This leads to a weighted Nash inequality if moreover $L V \leq c V$, whence to bounds such as (2.2).

A case study of symmetric semigroups on $\mathbb{R}$ consists in the Sturm-Liouville operators: given a probability measure $\mu$ with smooth and positive density $\rho$ with respect to the Lebesgue measure, the Sturm-Liouville operator

$$
L f=f^{\prime \prime}+\log (\rho)^{\prime} f^{\prime}
$$

defined on smooth functions leads to a symmetric Markov semigroup in $\mathcal{L}^{2}(\mu)$. Depending on $\rho$, this family shows all possible behaviours. The main example studied in this article concerns the probability measures

$$
d \mu_{a}(x)=\rho_{a}(x) d x=C_{a} e^{-|x|^{a}} d x
$$

on $\mathbb{R}$ and their associated Markov semigroup $\left(P_{t}\right)_{t \geq 0}$; here $a>0$ and $C_{a}$ is a normalization constant.

If $a>2$, the semigroup is ultracontractive and the density with respect to the measure $\mu_{a}$ is uniformly bounded (see [18] for the proof, among more general examples). In the limiting Gaussian case, when $a=2$, the semigroup is the well known Ornstein-Uhlenbeck semigroup (up to normalization), which is not ultracontractive but only hypercontractive. This means that, for $t>0, P_{t}$ maps $\mathcal{L}^{2}\left(\mu_{a}\right)$ 
into some $\mathcal{L}^{q(t)}\left(\mu_{a}\right)$, where $2<q(t)<\infty$ : this is Nelson's Theorem. Observe that in this case one knows explicitly the density $p_{t}(x, y)$ and the spectrum $\lambda_{n}=n$, and that $P_{t}$ is Hilbert-Schmidt.

If $1<a<2$, the semigroup $P_{t}$ is not hypercontractive since the measure $\mu_{a}$ does not satisfy a logarithmic Sobolev inequality. In fact, as shown in [7], $P_{t}$ with $t>0$ satisfies Orlicz hypercontractivity: it maps $\mathcal{L}^{2}\left(\mu_{a}\right)$ into a Orlicz space slightly smaller than $\mathcal{L}^{2}\left(\mu_{a}\right)$. This functional regularity does not yield any explicit upper bound on the kernel density $p_{t}$.

As a simple illustration of our general method, we shall prove that, for all real $\beta$, there exists $\theta>0$ such that the density $p_{t}(x, y)$ satisfies the explicit upper bound

$$
p_{t}(x, y) \leq C(a, \beta) \frac{e^{c t}}{t^{\theta}} \frac{\rho_{a}^{-1 / 2}(x) \rho_{a}^{-1 / 2}(y)}{\left(1+|x|^{2}\right)^{\beta}\left(1+|y|^{2}\right)^{\beta}} .
$$

For $\beta>1 / 2$, this estimate is in $\mathcal{L}^{2}\left(\mu_{a}\right)$, so that the operator $P_{t}$ is Hilbert-Schmidt. To our knowledge, this is a new result. In the other limiting case, when $a=1$, such an estimate cannot hold anymore. Indeed, the spectrum of $-L$ has not only a discrete part but lies in $\{0\} \cup\left(\lambda_{0}, \infty\right)$, with $\lambda_{0}>0$ (see [29]). Let us note that studying the measures $\mu_{a}$ for $a \in(1,2)$ is currently an active area in functional analysis. These measures represent a large class of log-concave measures: they are not log-concave enough to satisfy a logarithmic Sobolev inequality, but some of their properties, such as, for instance, concentration, are similar to those of the standard Gaussian measure. See, for example, [7], [8], [16], and [20].

The method used here to obtain the weighted Nash inequalities on the real line will be quite close to the method introduced by B. Muckenhoupt in [23] and generalized later by S. Bobkov and F. Götze in [11] to characterize measures which satisfy Poincaré or logarithmic Sobolev inequalities on the real line. We shall not try here to get the same kind of if and only if results, since there are too many parameters to control (the weight function $V$, the rate function $\Phi$, and so on).

We shall also not try to extend our results to the most general setting, for example Riemannian manifolds, as this would require a more precise analysis of the Laplacian of the distance function, and therefore lower bounds on the Ricci curvature. Instead we prefer to concentrate on some key one-dimensional models to show the ease and the efficiency of the methods presented here. Moreover, as it is typical when using Lyapunov functions, the constants obtained in these estimates are far from optimal, and that is why we only focus on the overall behavior of the estimates but do not try to get the best constants.

The plan of the article is the following. In the next section we explain the abstract result: how a weighted Nash inequality coupled to a Lyapunov function implies a non-uniform estimate of the kernel density. In Section 4 we prove a universal weighted Nash inequality. In Section 5 we finally apply the method of Section 3 to the measures $\mu_{a}$ defined above for $a \in(1,2)$.

Notation. In the whole article, $\|\cdot\|_{p}$ stands for the $\mathcal{L}^{p}$ norm with respect to the measure $\mu$. The measure $\mu$ could change, depending on the context, but it should be always clear. 


\section{The abstract result}

In this section we present a simple method to obtain the existence of and explicit and non-uniform bounds on Markov semigroup kernel densities.

In the classical ultracontractive case the upper bound on the kernel density $q^{2}$ of $Q \circ Q$ follows from

$$
\|Q f\|_{2} \leq\|f\|_{1} \Longleftrightarrow\|Q \circ Q f\|_{\infty} \leq\|f\|_{1} \Longleftrightarrow\left|q^{2}(x, y)\right| \leq 1 .
$$

We extend this property to non-uniform estimates.

Proposition 3.1. Let $(E, \mathcal{E}, \mu)$ be a nice measure space, $Q$ a symmetric bounded operator on $\mathcal{L}^{2}(\mu)$ and $V$ a positive measurable function on $E$. Then the two following assertions are equivalent:

(i) The operator $Q$ satisfies

$$
\|Q f\|_{2} \leq\|f V\|_{1}
$$

for all $f \in \mathcal{L}^{2}(\mu)$.

(ii) The operator $Q^{2}=Q \circ Q$ may be represented by a kernel density $q^{2}(x, y)$ with respect to $\mu$ which satisfies

$$
\left|q^{2}(x, y)\right| \leq V(x) V(y)
$$

for $\mu \otimes \mu$ almost every $(x, y)$ in $E \times E$.

If moreover the function $V$ is in $\mathcal{L}^{2}(\mu)$, then $Q$ is Hilbert-Schmidt, and therefore has a discrete spectrum $\left(\mu_{n}\right)_{n \in \mathbb{N}}$ such that

$$
\sum_{n} \mu_{n}^{2} \leq \int V^{2} d \mu .
$$

Proof. Let us assume (i) and let us consider the operator $Q_{1}=\frac{1}{V} Q V$, that is, defined by

$$
Q_{1} f=\frac{1}{V} Q(f V)
$$

By hypothesis, $Q_{1}$ is a contraction from $\mathcal{L}^{1}(\nu)$ into $\mathcal{L}^{2}(\nu)$ where $d \nu=V^{2} d \mu$. Moreover, it is symmetric with respect to the measure $\nu$, since $Q$ also is with respect to $\mu$. So, by duality, it is also a contraction from $\mathcal{L}^{2}(\nu)$ into $\mathcal{L}^{\infty}(\nu)$, and by composition the operator $Q_{1}^{2}=Q_{1} \circ Q_{1}$ is a contraction from $\mathcal{L}^{1}(\nu)$ into $\mathcal{L}^{\infty}(\nu)$.

This implies that $Q_{1}^{2}$ may be represented by a kernel density $q_{1}^{2}(x, y)$ in the space $\mathcal{L}^{2}(\nu)$ which satisfies $\left|q_{1}^{2}(x, y)\right| \leq 1$ for $\nu \otimes \nu$ almost every $(x, y)$ in $E \times E$ (see Lemme 4.3 in [2], for instance). On the other hand,

$$
q_{1}^{2}(x, y) V(x) V(y)=q^{2}(x, y)
$$

for $\mu \otimes \mu$ every $(x, y)$, noting that $V$ is positive. This implies (ii). 
Conversely, if $f \in \mathcal{L}^{2}(\mu)$, then, by symmetry of $Q$,

$$
\|Q f\|_{2}^{2}=\int f Q^{2} f d \mu=\int q^{2}(x, y) f(x) f(y) d(\mu \otimes \mu)(x, y) \leq\left(\int|f| V d \mu\right)^{2},
$$

which proves (i).

If now $V \in \mathcal{L}^{2}(\mu)$, then the kernel $q^{2}(x, x)$ is integrable on $E$ with respect to $\mu$, which just means that $Q$ is Hilbert-Schmidt.

Example 3.1. The first explicit example is the classical Ornstein-Uhlenbeck semigroup in $\mathbb{R}^{n}$, with generator $L=\Delta-x \cdot \nabla$. In a probabilistic form, it is given by the Mehler formula

$$
P_{t} f(x)=\mathbb{E}\left(f\left(e^{-t} x+\sqrt{1-e^{-2 t}} Y\right)\right),
$$

where $Y$ is a standard Gaussian variable with law $\gamma$. It admits a kernel density with respect to the Gaussian measure, given by

$$
p_{t}(x, y)=\left(1-e^{-2 t}\right)^{-n / 2} \exp \left(-\frac{1}{2\left(1-e^{-2 t}\right)}\left(|y|^{2} e^{-2 t}-2 x \cdot y e^{-t}+|x|^{2} e^{-2 t}\right)\right)
$$

for all $x, y \in \mathbb{R}^{n}$ and $t>0$. In particular,

$$
\begin{aligned}
p_{2 t}(x, y) & \leq p_{2 t}(x, x)^{1 / 2} p_{2 t}(y, y)^{1 / 2} \\
& =\left(1-e^{-4 t}\right)^{-n / 2} \exp \left(\frac{|x|^{2}}{1+e^{2 t}}\right) \exp \left(\frac{|y|^{2}}{1+e^{2 t}}\right)
\end{aligned}
$$

by the Cauchy-Schwarz inequality, with equality if $x=y$. Hence, by Proposition 3.1,

$$
\left\|P_{t} f\right\|_{L^{2}(d \gamma)} \leq\left\|f V_{t}\right\|_{L^{1}(d \gamma)}
$$

where

$$
V_{t}(y)=\left(1-e^{-4 t}\right)^{-n / 4} \exp \left(\frac{|y|^{2}}{2\left(1+e^{2 t}\right)}\right) \text {. }
$$

This bound has been obtained in a more general context in [4], where it is shown to be optimal, being an equality for square-exponential functions $f$.

Proposition 3.1 leads us to prove bounds such as (i).

When the operator $Q$ is a Markov semigroup $P_{t}$ with a kernel $p_{t}$, evaluated at time $t$, then one may obtain such bounds through functional inequalities that we describe here. We shall mainly be concerned with the case when $\mu$ is a probability measure, although much of what follows could be extended to the case where $\mu$ has infinite mass.

Let $\left(P_{t}\right)_{t \geq 0}$ be a symmetric Markov semigroup on $E$ with generator $L$ and associated Dirichlet form

$$
\mathcal{E}_{\mu}(f, f)=-\int f L f d \mu .
$$

This quadratic form can be defined on a larger subspace than the domain of $L$, which is called the domain of the Dirichlet form. 
Bounds such as $\left\|P_{t} f\right\|_{2} \leq K(t)\|f V\|_{1}$ will be obtained by means of weighted Nash inequalities and Lyapunov functions, that we now define.

Definition 3.2. Let $V$ be a positive function on $E$, let $M$ be a non-negative real number and let $\phi$ be a positive function defined on $(M, \infty)$ with $\phi(x) / x$ non decreasing.

The Dirichlet form $\mathcal{E}_{\mu}$ satisfies a weighted Nash inequality with weight $V$ and rate function $\phi$ if

$$
\phi\left(\frac{\|f\|_{2}^{2}}{\|f V\|_{1}^{2}}\right) \leq \frac{\mathcal{E}_{\mu}(f, f)}{\|f V\|_{1}^{2}}
$$

for all functions $f$ in the domain of the Dirichlet form such that

$$
\|f\|_{2}^{2}>M\|f V\|_{1}^{2} \text {. }
$$

As recalled in the introduction, the two fundamental examples are the classical Nash inequality (1.1) for the Lebesgue measure, with

$$
\phi(x)=C x^{1+2 / n} \quad \text { and } \quad M=0,
$$

$\left(M>b^{n / 2}\right.$ for the generalized inequality (1.4)), and those (1.8) given by superPoincaré inequalities, with $\phi$ the inverse of $\inf _{a}\{a x+b(a)\}$ and $M=0$. They all have weights $V=1$, and in the following we shall be concerned with Nash inequalities with a general positive weight $V$.

Definition 3.3. A Lyapunov function is a positive function $V$ on $E$ in the domain of the generator $L$ such that

$$
L V \leq c V
$$

for a real constant $c$, called the Lyapunov constant.

It is not really necessary for $V$ to be in the $\mathcal{L}^{2}$-domain of $L$, but for simplicity we restrict to this situation, which will be the situation in our examples below.

Remark 3.4. In our context, the Lyapunov constant $c$ will be non-negative. Negative Lyapunov constants can also be considered, but by adding an extra term. For instance, in [3] and [5], the authors consider Lyapunov functions $V$ such that

$$
L V \leq-\gamma V+\mathbf{1}_{K}
$$

where $\gamma>0, V \geq 1$ and $K$ is a compact set. These Lyapunov functions are a powerful tool for obtaining rates on the long time behavior of the Markov semigroup, for example, through obtaining Poincaré or more generally weak Poincaré inequalities.

As mentioned in the introduction, Lyapunov functions defined as in our Definition 3.3 with $c \geq 0$ are introduced to obtain smoothing properties of the Markov semigroup for a fixed time $t>0$.

When $\mu$ has finite mass, one can also observe that the restriction $V \geq 0$ in (3.3) could be replaced by $V \geq 1$ when $c \geq 0$, since one may always change $V$ into $V+1$. This will be the case in the main application given in Section 5 . 
Then, one has the following.

Theorem 3.5 (Wang). Let $\left(P_{t}\right)_{t \geq 0}$ be a Markov semigroup on E with generator $L$ symmetric in $\mathcal{L}^{2}(\mu)$.

Assume that there exists a Lyapunov function $V$ in $\mathcal{L}^{2}(\mu)$ with Lyapunov constant $c \geq 0$, and that the Dirichlet form associated to $L$ satisfies a weighted Nash inequality with weight $V$ and rate function $\phi$ on $(M,+\infty)$ such that

$$
\int^{\infty} \frac{1}{\phi(x)} d x<\infty
$$

Then

$$
\left\|P_{t} f\right\|_{2} \leq K(2 t) e^{c t}\|f V\|_{1}
$$

for all $t>0$ and all functions $f \in \mathcal{L}^{2}(\mu)$. Here, the function $K$ is defined by

$$
K(x)= \begin{cases}\sqrt{U^{-1}(x)} & \text { if } 0<x<U(M), \\ \sqrt{M} & \text { if } x \geq U(M)\end{cases}
$$

where $U$ denotes the (decreasing) function defined on $(M,+\infty)$ by

$$
U(x)=\int_{x}^{\infty} \frac{1}{\phi(u)} d u .
$$

We state and prove it in our context to show that our method is simple and self contained.

Here the measure $\mu$ need not be a probability measure and may have infinite mass and, in the case when $U(M)=+\infty$, then $K$ is just defined by the first line.

Observe also that if $M=0$ then we can take any real parameter $c$, as one can see from the proof.

Remark 3.6. As mentioned in Remark 3.4, we are not mainly concerned with the long time behaviour of the Markov semigroup, though in some cases a weighted Nash inequality may reveal adapted. For instance, in the case when $c=0, M=0$ and $U(M)=0$, then Theorem 3.5 ensures that $P_{t} f$ converges to 0 in $\mathcal{L}^{2}(\mu)$ for all $f \in \mathcal{L}^{2}(\mu)$ with finite $\|f V\|_{1}$. Observe that in this case $\mu$ has necessarily infinite mass. If $\mu$ is a probability measure, then we expect $P_{t} f$ to converge to $\int f d \mu$, which is a priori nonzero, so the rate $K(2 t) e^{c t}$ cannot converge to 0 .

On the contrary, weighted Nash inequalities are adapted to get estimates on the small time behavior: Theorem 3.5 gives a bound on $\left\|P_{t} f\right\|_{2}$ for $t>0$ which depends on $f$ only in terms of a weighted $\mathcal{L}^{1}$ norm, which is an illustration of the gain of integrability induced by the semigroup. Observe that the coefficient $K(2 t)$ tends to $+\infty$ as $t$ goes to 0 .

By Proposition 3.1 this leads to the following bounds on the kernels:

Corollary 3.7. If the Markov semigroup $\left(P_{t}\right)_{t \geq 0}$ satisfies the assumptions of Theorem 3.5 above, then $P_{t}$ has a density $p_{t}$ with respect to $\mu$ which satisfies

$$
p_{2 t}(x, y) \leq K(2 t)^{2} e^{2 c t} V(x) V(y),
$$

for all $t>0$ and $\mu \otimes \mu$ almost every $(x, y) \in E \times E$. 
Moreover, $P_{t}$ is Hilbert-Schmidt for all $t>0$, and therefore has a discrete spectrum $\left(\mu_{n}(t)\right)_{n \in \mathbb{N}}$ such that

$$
\sum_{n} \mu_{n}(t)^{2} \leq K(2 t)^{2} e^{2 c t} \int V^{2} d \mu
$$

Proof of Theorem 3.5. Let $f$ in $\mathcal{L}^{2}(\mu)$ be given. With no loss of generality we can assume that $f>0$ by writing the argument for $|f|+\varepsilon$, and letting $\varepsilon$ go to 0 and using the bound $\left|P_{t} f\right| \leq P_{t}|f|$.

First notice that the map $G(t)=\int V P_{t} f d \mu$ has derivative

$$
G^{\prime}(t)=\int V L P_{t} f d \mu=\int L V P_{t} f d \mu \leq c G(t)
$$

so that

$$
\int V P_{t} f d \mu \leq e^{c t} \int V f d \mu .
$$

Then, given $0 \leq t \leq T$ fixed, consider the function

$$
R(s)=\frac{\left\|P_{s} f\right\|_{2}^{2}}{\left(e^{c t} \int f V d \mu\right)^{2}}
$$

on $[0, t]$. Then

$$
\frac{-R^{\prime}(s)}{2}=\frac{\mathcal{E}_{\mu}\left(P_{s} f, P_{s} f\right)}{\left(e^{c t} \int f V d \mu\right)^{2}}=\frac{\mathcal{E}_{\mu}\left(P_{s} f, P_{s} f\right)}{\left(\int P_{s} f V d \mu\right)^{2}}\left(\frac{\int P_{s} f V d \mu}{e^{c t} \int f V d \mu}\right)^{2} .
$$

In particular $R$ is decreasing. Moreover, if there exists $s \in[0, t]$ such that $R(s) \leq M$, then $R(t) \leq R(s) \leq M$, which yields the result. Hence we now assume that $R(s) \geq M$ on $[0, t]$. Then, by $(3.5)$,

$$
\frac{\left\|P_{s} f\right\|_{2}^{2}}{\left(\int P_{s} f V d \mu\right)^{2}}=\frac{\left\|P_{s} f\right\|_{2}^{2}}{\left(e^{c t} \int f V d \mu\right)^{2}} \frac{\left(e^{c t} \int f V d \mu\right)^{2}}{\left(\int P_{s} f V d \mu\right)^{2}}=R(s) e^{2 c(t-s)} \frac{\left(e^{c s} \int f V d \mu\right)^{2}}{\left(\int P_{s} f V d \mu\right)^{2}} \geq M
$$

for $c \geq 0$.

Hence, by applying the weighted Nash inequality to $P_{s} f,(3.6)$ gives

$$
\frac{-R^{\prime}(s)}{2} \geq \phi\left(\frac{\left\|P_{s} f\right\|_{2}^{2}}{\left(\int P_{s} f V d \mu\right)^{2}}\right)\left(\frac{\int P_{s} f V d \mu}{e^{c t} \int f V d \mu}\right)^{2} .
$$

Moreover,

$$
\phi\left(\frac{\left\|P_{s} f\right\|_{2}^{2}}{\left(\int P_{s} f V d \mu\right)^{2}}\right) \geq \phi\left(\frac{\left\|P_{s} f\right\|_{2}^{2}}{\left(e^{c t} \int f V d \mu\right)^{2}}\right)\left(\frac{\int P_{s} f V d \mu}{e^{c t} \int f V d \mu}\right)^{2}
$$


from the inequality (3.5) and the fact that $\phi(x) / x$ is non decreasing, so that

$$
\frac{-R^{\prime}(s)}{2} \geq \phi(R(s))
$$

In turn this may be seen as

$$
U(R(s))^{\prime} \geq 2
$$

which integrates to

$$
U(R(t)) \geq U(R(0))+2 t \geq 2 t .
$$

Since $U^{-1}$ is defined on $(0, U(M)]$ and is decreasing, we obtain the upper bound

$$
R(t) \leq U^{-1}(2 t)
$$

for all $t \leq U(M) / 2$. For $t \geq U(M) / 2$, we have $R(t) \leq M$. Combining all these estimates gives the result.

Remark 3.8. In the main application of the weighted Nash inequality given in Section 5, the weight function $V$ is in $\mathcal{L}^{2}(\mu)$. However, formally one does not need $V$ to be in $\mathcal{L}^{2}(\mu)$ to get the result. This restriction is made here not only in view of Proposition 3.1. It is also made to ensure the integration by parts formula

$$
\int L P_{s} f V d \mu=\int P_{s} f L V d \mu
$$

which leads to (3.5), and automatically holds when $V$ is in $\mathcal{L}^{2}(\mu)$ and in the domain of $L$. For those $V$ which increase too rapidly at infinity, this may be false in general; it requires a more precise analysis of the semigroup $\left(P_{t}\right)_{t \geq 0}$ and restricting to a large subclass of functions in $\mathcal{L}^{2}(\mu)$.

Here are two fundamental examples in the two cases when $\mu$ has finite or infinite mass:

- The Lebesgue measure on $\mathbb{R}^{n}$ satisfies the classical Nash inequality (1.1), hence a weighted Nash inequality with weight $V=1$ and rate function $\phi(x)=$ $C x^{1+2 / n}$, for instance on the set $(0,+\infty)$. Then, by Theorem 3.5 applied with $V=1$ and $c=0$, one recovers the well known contraction property of the classical heat kernel on $\mathbb{R}^{n}$,

$$
\left\|P_{t} f\right\|_{2} \leq\left(\frac{C}{t}\right)^{n / 4}\|f\|_{1}
$$

for all $t>0$ and for the non-optimal constant $C=n / 4$ instead of $1 /(8 \pi)$ (see [21], for instance). In this case, we only have to consider functions $f \in \mathcal{L}^{1}(\mu)$ and in the domain of the Dirichlet form $\int|\nabla f|^{2} d \mu$. The main tool to get optimal bounds in $\mathcal{L}^{p}(\mu)$ for any $p \geq 1$ for the classical heat kernel on $\mathbb{R}^{n}$ is the Euclidean logarithmic Sobolev inequality, as explained for instance in [2] or [21]. 
- The second example concerns the Sturm-Liouville operator $L f=f^{\prime \prime}+$ $(\log \rho)^{\prime} f^{\prime}$ on $\mathbb{R}$, associated with the measure $d \mu=\rho(x) d x$. Here it would be enough to know that $(\log \rho)^{\prime \prime}$ is bounded from above and that $V \rho^{\prime}$ and $V^{\prime} \rho$ go to 0 at infinity. Indeed, in this situation, it is enough for smooth functions $f$ and $g$ that $f^{\prime} g \rho$ and $f g^{\prime} \rho$ go to 0 at infinity to ensure, through integration by parts, that

$$
\int L f g d \mu=-\int f^{\prime} g^{\prime} d \mu=\int f L g d \mu .
$$

When $(\log \rho)^{\prime \prime}$ is bounded from above, the semigroup satisfies a $C D(a, \infty)$ inequality. Hence, when $f$ is bounded, then so is $\left(P_{t} f\right)^{\prime}$ when $t>0$ (see Remark 5.4.2 in [1]). Hence in this case we may work with the space of bounded functions to get the result.

Examples will be studied in Sections 4 and 5 .

Theorem 3.5 has the following converse:

Theorem 3.9. Let $\mu$ be a measure on $E$ and let $\left(P_{t}\right)_{t \geq 0}$ be a Markov semigroup on $E$ with generator $L$ symmetric in $\mathcal{L}^{2}(\mu)$.

If there exists a positive function $V$ and a positive function $K$ defined on $(0, \infty)$ such that

$$
\left\|P_{t} f\right\|_{2} \leq K(t)\|f V\|_{1}
$$

for all $t>0$, then the weighted Nash inequality (3.2) holds with the same function $V, M=0$ and the function

$$
\phi(x)=\sup _{t>0} \frac{x}{2 t} \log \frac{x}{K(t)^{2}}, \quad x \geq 0 .
$$

Here, again, $\mu$ need not be a probability measure.

Remark 3.10. For instance, by Theorem 3.5, if we assume a Nash inequality with $\phi(x)=C x^{r}$ for large $x$, with $r>1$, then we obtain a bound such as $\left\|P_{t} f\right\|_{2} \leq$ $K(t)\|f V\|_{1}$ with $K(t)=C^{\prime} t^{1 / 2(1-r)}$ for small $t$.

Conversely, if we assume such a bound with such a $K$, then, by the converse Theorem 3.9, we obtain a Nash inequality with function $\phi(x)=C^{\prime \prime} x^{r}$ for large $x$. Therefore, in this case and up to the values of the constants, we have a true quantitative equivalence between the Nash inequality and the bound on $\left\|P_{t} f\right\|_{2}$.

Proof of Theorem 3.9. It is based on the observation that the function

$$
t \mapsto \log \left(\left\|P_{t} f\right\|_{2}^{2}\right)
$$

is convex for any symmetric semigroup. Indeed, if $h(t)=\left\|P_{t} f\right\|^{2}$, then $h^{\prime}(t)=$ $2 \int P_{t} f L\left(P_{t} f\right) d \mu$ and $h^{\prime \prime}(t)=4 \int\left(L P_{t} f\right)^{2} d \mu$; hence $h^{\prime 2} \leq h h^{\prime \prime}$, or equivalently $(\log h)^{\prime \prime} \geq 0$.

Therefore,

$$
\log h(u)-\log h(0) \leq \frac{u}{t}[\log h(t)-\log h(0)]
$$


for all $0<u \leq t$, so that

$$
h^{\prime}(0) \leq \frac{h(0)}{t} \log \frac{h(t)}{h(0)}
$$

by letting $u$ go to 0 .

Now, if moreover

$$
h(t) \leq K(t)^{2}\|f V\|_{1}^{2}
$$

then (3.7) gives

$$
-2 \frac{\mathcal{E}(f, f)}{\|f V\|_{1}^{2}} \leq \frac{\|f\|_{2}^{2}}{\|f V\|_{1}^{2}} \frac{1}{t} \log \left(\frac{K(t)^{2}\|f V\|_{1}^{2}}{\|f\|_{2}^{2}}\right) .
$$

This gives the claimed weighted Nash inequality.

\section{A universal weighted Nash inequality on $\mathbb{R}^{n}$}

Let $\rho$ be a positive smooth function on $\mathbb{R}^{n}$. We prove a weighted Nash inequality for the operator $L f=\Delta f+\nabla \log \rho \cdot \nabla f$ with the universal weight $V=\rho^{-1 / 2}$ and the measure $d \mu(x)=\rho(x) d x$. As usual, $\|\cdot\|_{p}$ stands for the $\mathcal{L}^{p}(\mu)$ norm and $\left(P_{t}\right)_{t \geq 0}$ is the semigroup with generator $L$.

Theorem 4.1. In the above notation, the classical Nash inequality (1.1) is equivalent to

$$
\|f\|_{2}^{2+\frac{4}{n}} \leq C_{n}^{\frac{4}{n}}\|f V\|_{1}^{\frac{4}{n}}\left(\mathcal{E}(f, f)+\int_{\mathbb{R}^{n}} \frac{L V}{V} f^{2} d \mu\right)
$$

for all smooth functions $f$ on $\mathbb{R}^{n}$ with compact support. If moreover $L V \leq c V$ for $c \in \mathbb{R}$, then

$$
\|f\|_{2}^{2+\frac{4}{n}} \leq C_{n}^{\frac{4}{n}}\|f V\|_{1}^{\frac{4}{n}}\left(\mathcal{E}(f, f)+c \int_{\mathbb{R}^{n}} f^{2} d \mu\right)
$$

Proof. Let $g$ be a smooth function with compact support and let $f=g \sqrt{\rho}$. Then

$$
\begin{aligned}
\int_{\mathbb{R}^{n}}|f|^{2} d x & =\|g\|_{2}^{2}, \\
\int_{\mathbb{R}^{n}}|f| d x & =\int_{\mathbb{R}^{n}}|g| \sqrt{\rho} d x=\|g V\|_{1},
\end{aligned}
$$

and

$$
\int_{\mathbb{R}^{n}}|\nabla f|^{2} d x=\int_{\mathbb{R}^{n}}|\nabla g|^{2} d \mu+\int_{\mathbb{R}^{n}} 2 \frac{g}{V} \nabla g \cdot \nabla \frac{1}{V} d x+\int_{\mathbb{R}^{n}} g^{2}\left|\nabla\left(\frac{1}{V}\right)\right|^{2} d x .
$$

By integration by part, the middle term is

$$
\int_{\mathbb{R}^{n}} \nabla\left(g^{2}\right) \cdot\left(\frac{1}{V} \nabla \frac{1}{V}\right) d x=-\int_{\mathbb{R}^{n}} g^{2} \nabla\left(\frac{1}{V} \nabla \frac{1}{V}\right) d x=\int_{\mathbb{R}^{n}} g^{2}\left(\frac{\Delta V}{V}-3 \frac{|\nabla V|^{2}}{V^{2}}\right) d \mu,
$$


so that

$$
\int_{\mathbb{R}^{n}}|\nabla f|^{2} d x=\int_{\mathbb{R}^{n}}|\nabla g|^{2} d \mu+\int_{\mathbb{R}^{n}} g^{2}\left(\frac{\Delta V}{V}-2 \frac{|\nabla V|^{2}}{V^{2}}\right) d \mu .
$$

Moreover

$$
\frac{L V}{V}=\frac{1}{V}(\Delta V-2 \nabla \log V \cdot \nabla V)=\frac{\Delta V}{V}-2 \frac{|\nabla V|^{2}}{V^{2}}
$$

so

$$
\int_{\mathbb{R}^{n}}|\nabla f|^{2} d x=\mathcal{E}(g, g)+\int_{\mathbb{R}^{n}} \frac{L V}{V} g^{2} d \mu .
$$

Hence the classical Nash inequality (1.1) for $f$ is equivalent to (4.1) for $g$, which concludes the proof.

This type of transformation has been made by F.-Y. Wang in [28] at the level of the super-Poincaré inequality (1.7). From this, the author estimates the kernel densities of semigroups with infinite invariant measures. Using Theorem 4.1 we now give estimates in the case of invariant probability measures.

Corollary 4.2. With the above notation, assume that $\mu$ is a probability measure and that $V \in \mathcal{L}^{1}(\mu)$ satisfies $L V \in \mathcal{L}^{1}(\mu)$ and $L V \leq c V$ with $c \geq 0$. Assume moreover that the Hessian of $\log \rho$ is uniformly bounded from above on $\mathbb{R}^{n}$ and that

$$
\sup _{|x|=r} \rho(x)^{1 / 2} r^{n-1} \rightarrow 0 \quad \text { and } \quad \sup _{|x|=r}|\nabla \rho(x)| \rho^{-1 / 2} r^{n-1} \rightarrow 0
$$

as $r$ tends to infinity. Then $P_{t}$ has a density $p_{t}$ which satisfies

$$
p_{2 t}(x, y) \leq \frac{d}{t^{n / 2}} e^{2 c t} V(x) V(y)
$$

for some $d>0$ and for all $x, y \in \mathbb{R}^{n}, t>0$.

Proof. We cannot apply Theorem 3.5 directly since $V=\rho^{-1 / 2}$ is never in $\mathcal{L}^{2}(\mu)$. The argument is exactly the same, but we have to justify the inequality $G^{\prime}(t) \leq$ $c G(t)$ where $G(t)=\int V P_{t} f d \mu$ for any smooth function $f$ with compact support.

First of all $G^{\prime}(t)=\int V L P_{t} f d \mu$ since $V \in \mathcal{L}^{1}(\mu)$ and $L f$ is bounded.

Then we prove the integration by parts

$$
\int_{\mathbb{R}^{n}} V L P_{t} f d \mu=\int_{\mathbb{R}^{n}} L V P_{t} f d \mu .
$$

Let $r>0$, let $B_{r}$ be the centered ball of $\mathbb{R}^{n}$ with radius $r$ and let $\vec{v}$ be its outward unit normal vector. Then, by two integrations by parts on $B_{r}$,

$$
\begin{aligned}
\int_{B_{r}} V L P_{t} f d \mu= & \int_{B_{r}} L V P_{t} f d \mu-\int_{S^{n-1}} P_{t} f(r \omega) \nabla V(r \omega) \cdot \vec{v} \rho(r \omega) r^{n-1} d \omega \\
& +\int_{S^{n-1}} V(r \omega) \nabla P_{t} f(r \omega) \cdot \vec{v} \rho(r \omega) r^{n-1} d \omega .
\end{aligned}
$$


However, the Hessian of $\log \rho$ is uniformly bounded from above on $\mathbb{R}^{n}$, say by the real number $\lambda$, so $L$ satisfies a $C D(-\lambda, \infty)$ curvature-dimension criterion. In particular (see [2] for instance), this implies the uniform bound

$$
\left|\nabla P_{t} f\right| \leq e^{\lambda t} P_{t}|\nabla f| \leq e^{\lambda t}\|\nabla f\|_{\infty} .
$$

Then our assumptions on $\rho$ ensure that the last two terms tend to 0 as $r$ tends to infinity, which justifies the integration by parts.

Remark 4.3. The key point here is that $V=\rho^{-1 / 2}$ is never in $\mathcal{L}^{2}(\mu)$, so this does not resolve whether $P_{t}$ is Hilbert-Schmidt or not.

We illustrate Corollary 4.2 on the examples of measures of Cauchy and exponential type. We have in mind the measure $\exp \left(-|x|^{a}\right) d x$ in $\mathbb{R}^{n}$, but for convenience we will study $\exp \left(-\left(1+|x|^{2}\right)^{a / 2}\right) d x$ instead of $\exp \left(-|x|^{a}\right) d x$, as it has the same behavior at infinity and has no singularity at $x=0$.

Corollary 4.4. Let $\rho(x)=\left(1+|x|^{2}\right)^{-\beta}$ with $\beta>n$ or $\rho(x)=\exp \left(-\left(1+|x|^{2}\right)^{a / 2}\right)$ with $a>0$. Then there exists a constant $C$ such that for all $t>0$ and $x, y \in \mathbb{R}^{n}$ the kernel density $p_{t}$ satisfies

$$
p_{t}(x, y) \leq \frac{C}{t^{n / 2}} e^{C t} \rho^{-1 / 2}(x) \rho^{-1 / 2}(y) .
$$

In the next section we shall improve the bound on the kernel density in the case of the measure with density $\rho(x)=\exp \left(-\left(1+|x|^{2}\right)^{a / 2}\right)$ with $a>1$. For that purpose we shall use a Lyapunov function $V$ which will be now in $\mathcal{L}^{2}(\mu)$.

\section{The measures on $\mathbb{R}$ between exponential and Gaussian}

In this section we shall prove that the weighted Nash inequality (3.2) holds with power functions $\phi$ and $\mathcal{L}^{2}$ weights $V$ for the semigroups on $\mathbb{R}$ with the invariant measure $\exp \left(-|x|^{a}\right) d x$. Again for convenience we will study $\exp \left(-\left(1+x^{2}\right)^{a / 2}\right) d x$ instead of $\exp \left(-|x|^{a}\right) d x$.

For the analysis made here, it would make no difference if one were to work on $\mathbb{R}^{n}$, except for the values of the involved constants. We shall let

$$
T(x)=\left(1+x^{2}\right)^{1 / 2},
$$

and for $a>0$ define the probability measure

$$
d \mu_{a}=C_{a} e^{-T^{a}} d x
$$

where $C_{a}$ is the normalizing constant.

We are dealing with the Sturm-Liouville operator

$$
L f=f^{\prime \prime}-a T^{a-1} T^{\prime} f^{\prime},
$$

which is symmetric (and even self adjoint) with respect to the probability measure $\mu_{a}$. 
We let $\rho_{a}$ denote the density function of the measure $\mu_{a}$ with respect to the Lebesgue measure, that is

$$
\rho_{a}=\exp \left(-T^{a}\right)
$$

In this case, $f$ is in the domain of the Dirichlet form as soon as $f^{\prime} \in \mathcal{L}^{2}\left(\mu_{a}\right)$ and

$$
\mathcal{E}_{\mu_{a}}(f, f)=\int f^{\prime 2} d \mu_{a}=-\int f L f d \mu_{a}
$$

We shall not pay too much attention to the values of the constants, which may be far from optimal.

Lemma 5.1. For all $a>0$ and $\beta \in \mathbb{R}$ the function

$$
V=\rho_{a}^{-1 / 2} T^{-\beta}=\exp \left(\frac{T^{a}}{2}\right) T^{-\beta}
$$

is a Lyapunov function; moreover $V \in \mathcal{L}^{2}\left(\mu_{a}\right)$ as soon as $\beta>1 / 2$.

Proof. First observe that $V$ is positive, and that it is a Lyapunov function with constant $c$ if and only if

$$
L(\log V)+(\log V)^{\prime 2} \leq c .
$$

However, with $T=T(x)$,

$$
\begin{aligned}
& L(\log V)+(\log V)^{\prime 2} \\
& \quad=\frac{a}{2}(a-1) T^{a-2} T^{\prime 2}-\frac{a^{2}}{4} T^{2 a-2} T^{\prime 2}+\beta(\beta+1) \frac{T^{\prime 2}}{T^{2}}+\frac{a}{2} T^{a-1} T^{\prime \prime}-\beta \frac{T^{\prime \prime}}{T} \\
& \quad=\frac{a}{4} T^{a-4}\left(2(a-1) x^{2}-a T^{a} x^{2}+2\right)+\beta(\beta+1) x^{2} T^{-4}-\beta T^{-4}
\end{aligned}
$$

since $T^{\prime}(x)=x T(x)^{-1}$ and $T^{\prime \prime}=T(x)^{-3}$. Now for all $a>0$ the bracket is nonnegative at infinity and for all $\beta$ the last two terms go to 0 , so the continuous map $L(\log V)+(\log V)^{\prime 2}$ is bounded from above on $\mathbb{R}$.

The first basic result is the following:

Lemma 5.2. For all $a \geq 1$ and $\beta>0$ there exists a constant $C=C(a, \beta)$ such that, for all smooth and compactly supported functions $f$ such that $f(0)=0$,
i) $\int f^{2} d \mu_{a} \leq C \mathcal{E}_{\mu_{a}}(f, f)$
ii) $\int f^{2} d \mu_{a} \leq C \mathcal{E}_{\mu_{a}}(f, f)^{\gamma}\left(\int|f| V d \mu_{a}\right)^{2(1-\gamma)}$

where $V$ is the weight given by (5.1) and $\gamma=1-2 \frac{a-1}{3(a-1)+2 \beta} \in\left(\frac{1}{3}, 1\right]$. 
Proof. We shall let $C$ denote diverse constants depending only on $a$ in the proof of (i), and only on $a$ and $\beta$ in the proof of (ii).

For $x>0$ we let $q(x)=\int_{x}^{\infty} d \mu_{a}(y)$. The argument will be based on the following classical estimate (see for instance Corollaire 6.4.2 in [1]):

$$
q(x) \leq C \frac{\rho_{a}(x)}{T(x)^{a-1}} .
$$

To prove (i), and for $f$ satisfying $f(0)=0$, we write

$$
\int_{0}^{\infty} f^{2} d \mu_{a}=2 \iint_{t=0}^{x} f(t) f^{\prime}(t) d \mu_{a}(x) d t=2 \int_{0}^{\infty} f(t) f^{\prime}(t) q(t) d t .
$$

However, by (5.2), we have the upper bound $q(t) \leq C \rho_{a}(t)$ since $a, T \geq 1$, so that

$$
\int_{0}^{\infty} f^{2} d \mu_{a} \leq C\|f\|_{2} \mathcal{E}_{\mu_{a}}(f, f)^{1 / 2}
$$

by the Cauchy-Schwarz inequality. A similar result holds for the integral on $(-\infty, 0]$, which gives (i).

Let us now prove (ii) for $a>1$, since for $a=1$ it amounts to (i). Without loss of generality, we assume that $f$ is non-negative. Then

$$
\int_{0}^{\infty} f^{2} d \mu_{a}=\int_{0}^{\infty} f^{2} \mathbf{1}_{\left\{\frac{f}{\pi f \pi_{2}} \leq V Z^{-1 / 2}\right\}} d \mu_{a}+\int_{0}^{\infty} f^{2} \mathbf{1}_{\left\{\frac{f}{\pi f \pi_{2}}>V Z^{-1 / 2}\right\}} d \mu_{a}
$$

where $Z$ is a positive constant to be chosen later on. The first term is bounded from above by $\|f\|_{2} Z^{-1 / 2} \int f V d \mu_{a}$. Then we write the second one as

$$
\begin{aligned}
& \int_{0}^{\infty} f^{2} \mathbf{1}_{\left\{\frac{f}{\pi f \|_{2}}>V Z^{-1 / 2}\right\}} d \mu_{a} \\
&=2 \int_{0}^{\infty} f(t) f^{\prime}(t)\left[\int_{t}^{\infty} \mathbf{1}_{\left\{\frac{f(x)}{\|f\|_{2}}>V(x) Z^{-1 / 2}\right\}} d \mu_{a}(x)\right] d t
\end{aligned}
$$

by writing $f^{2}(x)=2 \int_{0}^{x} f(t) f^{\prime}(t) d t$. We bound the inner integral in the following two ways.

On the one hand, by (5.2),

$$
\int_{t}^{\infty} \mathbf{1}_{\left\{\frac{f(x)}{\|f\|_{2}}>V(x) Z^{-1 / 2}\right\}} d \mu_{a}(x) \leq \int_{t}^{\infty} d \mu_{a}(x)=q(t) \leq C \rho_{a}(t) T(t)^{1-a}
$$

On the other hand the map $y \mapsto e^{y / 2} y^{-\beta}$ is decreasing on $(0,2 \beta]$ and then increasing, and $T \geq 1$. Hence, $V$ is increasing on $(0,+\infty)$ if $2 \beta \leq 1$, and it is decreasing on $\left(0, \sqrt{4 \beta^{2}-1}\right]$ and then increasing otherwise. In any case, there exists $C$ such that $V(x) \geq C V(t)$ for all $x \geq t>0$. Hence,

$$
\begin{aligned}
\int_{t}^{\infty} \mathbf{1}_{\left\{\frac{f(x)}{\|f\|_{2}}>V(x) Z^{-1 / 2}\right\}} d \mu_{a}(x) & \leq \int_{t}^{\infty} \mathbf{1}_{\left\{\frac{f(x)}{\|f\|_{2}}>C V(t) Z^{-1 / 2}\right\}} d \mu_{a}(x) \\
& \leq \frac{Z}{C^{2} V^{2}(t)}=\frac{Z}{C^{2}} \rho_{a}(t) T(t)^{2 \beta}
\end{aligned}
$$

by the Markov inequality. 
Therefore,

$$
\int_{t}^{\infty} \mathbf{1}_{\left\{\frac{f(x)}{\|f\|_{2}}>V(x) Z^{-1 / 2}\right\}} d \mu_{a}(x) \leq C \rho_{a}(t) \min \left\{T(t)^{1-a}, T^{2 \beta}(t) Z\right\} .
$$

Now, since $a+2 \beta-1>0$ and $T$ is increasing, for any $Z \in(0,1]$ there exists $t_{0}$ such that $T\left(t_{0}\right)^{a+2 \beta-1}=1 / Z$, that is, $T\left(t_{0}\right)^{1-a}=T\left(t_{0}\right)^{2 \beta} Z$. We split the integral in (5.3) into two parts, according to wether $t \geq t_{0}$ or not, and obtain

$$
\begin{aligned}
\int_{0}^{\infty} f^{2} \mathbf{1}_{\left\{\frac{f}{\|f\|_{2}}>V Z^{-1 / 2}\right\}} d \mu_{a} & \leq C Z \int_{0}^{t_{0}}\left|f f^{\prime}\right| T^{2 \beta} d \mu_{a}+C \int_{t_{0}}^{\infty}\left|f f^{\prime}\right| T^{1-a} d \mu_{a} \\
& \leq C Z T^{2 \beta}\left(t_{0}\right) \int_{0}^{t_{0}}\left|f f^{\prime}\right| d \mu_{a}+C T^{1-a}\left(t_{0}\right) \int_{t_{0}}^{\infty}\left|f f^{\prime}\right| d \mu_{a}
\end{aligned}
$$

since $\beta>0$ and $1-a<0$. Moreover $Z T^{2 \beta}\left(t_{0}\right)=T\left(t_{0}\right)^{1-a}$, so

$$
\int_{0}^{\infty} f^{2} \mathbf{1}_{\left\{\frac{f}{\|f\|_{2}}>V Z^{-1 / 2}\right\}} d \mu_{a} \leq C T\left(t_{0}\right)^{1-a}\|f\|_{2} \mathcal{E}_{\mu_{a}}(f, f)^{1 / 2}
$$

by the Cauchy-Schwarz inequality.

In the end we have obtained the bound

$$
\|f\|_{2} \leq C\left(Z^{-1 / 2} \int f V d \mu_{a}+Z^{\frac{1-a}{1-a-2 \beta}} \mathcal{E}_{\mu_{a}}(f, f)^{1 / 2}\right)
$$

for all $0<Z \leq 1$.

If $\int f V d \mu_{a} \leq \mathcal{E}_{\mu_{a}}(f, f)^{1 / 2}$, we choose

$$
Z=\left(\frac{\int f V d \mu_{a}}{\mathcal{E}_{\mu_{a}}(f, f)^{1 / 2}}\right)^{\frac{2(1-a-2 \beta)}{3(1-a)-2 \beta}} \in(0,1]
$$

to get the inequality

$$
\int_{0}^{\infty} f^{2} d \mu_{a} \leq C \mathcal{E}_{\mu_{a}}(f, f)^{\gamma}\left(\int f V d \mu_{a}\right)^{2(1-\gamma)}
$$

where $\gamma=(a-1+2 \beta) /(3(a-1)+2 \beta)$. The same estimate holds on $(-\infty, 0]$, which gives (ii).

If now $\mathcal{E}_{\mu_{a}}(f, f)^{1 / 2} \leq \int f V d \mu_{a}$, then, by (i),

$$
\begin{aligned}
\int f^{2} d \mu_{a} \leq C \mathcal{E}_{\mu_{a}}(f, f) & =C \mathcal{E}_{\mu_{a}}(f, f)^{\gamma} \mathcal{E}_{\mu_{a}}(f, f)^{1-\gamma} \\
& \leq C \mathcal{E}_{\mu_{a}}(f, f)^{\gamma}\left(\int f V d \mu_{a}\right)^{2(1-\gamma)}
\end{aligned}
$$

for all $0 \leq \gamma \leq 1$, which gives (ii). 
Remark 5.3. The first inequality of Lemma 5.2 is only based on the tail estimate $q(t) \leq C \rho_{a}(t)$, so holds for all measures $d \mu=\rho d x$ such that $q(x) \leq C \rho(x)$, where $q(x)=\mu([x,+\infty))$. In particular such probability measures $\mu$ satisfy a spectral gap inequality

$$
\|f\|_{2}^{2} \leq\left(\int f d \mu\right)^{2}+C \mathcal{E}_{\mu}(f, f)
$$

by applying (i) to $f-f(0)$, since

$$
\operatorname{Var}_{\mu}(f):=\int f^{2} d \mu-\left(\int f d \mu\right)^{2} \leq \int(f-c)^{2} d \mu
$$

for all constants $c$, and in particular for $c=f(0)$.

In fact the probability measure $\mu_{a}$ is log-concave on $\mathbb{R}$ and, according to the Bobkov Theorem (see [10]), all log-concave measures on $\mathbb{R}^{n}$ satisfy a Poincaré inequality. Note that a proof of this result is given in [3] by using the Lyapunov function $W=e^{\gamma T^{a}}$ for a $\gamma>0$.

Remark 5.4. The condition $a \geq 1$ is crucial in this proof of Lemma 5.2. The second inequality is obtained for all $\beta>0$. For $\beta \leq 0$ we may use the bound (5.5) with $T(t)^{2 \beta} \leq 1$, but not the bound (5.4); then we choose $Z=\left(\int f V d \mu_{a} \mathcal{E}_{\mu_{a}}(f, f)^{-1 / 2}\right)^{2 / 3}$ to obtain (ii) with $\gamma=1 / 3$. Observe that the best bound is obtained for $\beta=0$, for which we have the following general bound.

Remark 5.5. Let $\mu$ be a probability measure on $\mathbb{R}$, with a density $\rho(x)$ increasing on $(-\infty, 0)$ and decreasing on $(0, \infty)$ and let $V=\rho^{-1 / 2}$. Then

$$
\|f\|_{2} \leq\left(\frac{27}{2}\right)^{1 / 3}\left(\int|f| V d \mu\right)^{1 / 3} \mathcal{E}_{\mu}(f, f)^{1 / 3}
$$

for all smooth functions such that $f(0)=0$. The proof follows the argument of Lemma 5.2 , by using the bound (5.5) but not (5.4). This gives a Nash inequality with $\phi(x)=2 x^{3 / 2} / 27$ on $(0,+\infty)$, so that $1 / \phi$ is integrable at infinity. However, besides the restriction $f(0)=0$ which will be removed below only for $a>3$ (with $\beta=0$ ), it does not give an upper bound on the density, as in Corollary 3.7, since $V$ is not in $\mathcal{L}^{2}(\mu)$.

The restriction $f(0)=0$ is removed by the following:

Lemma 5.6. Given the measure $d \mu_{a}=C_{a} \exp \left(-T^{a}\right) d x$ with $a>0$ and the weight function

$$
V=\exp \left(T^{a} / 2\right) T^{-\beta} \quad \text { with } \quad \beta>\frac{3-a}{2},
$$

then there exist $\theta \in(0,1)$ and constant $C$ such that

$$
\int|f-f(0)| V d \mu_{a} \leq C\left[\int|f| V d \mu_{a}+\left(\int|f| V d \mu_{a}\right)^{1-\theta} \mathcal{E}_{\mu_{a}}(f, f)^{\theta / 2}\right]
$$

for all non-negative smooth compactly supported $f$ on $\mathbb{R}$.

Remark 5.7. For $\beta>3 / 2$ then all $\theta \in(2 / 3,1)$ are admissible. 
Proof. In the proof we shall let $C$ denote diverse constants which depend only on $a, \beta$ and a parameter $\alpha$ to be introduced later. We start by writing

$$
\int|f-f(0)| V d \mu_{a} \leq \int|f| V d \mu_{a}+|f(0)| \int V d \mu_{a}
$$

For convenience we let

$$
U=\int|f| V d \mu
$$

For any $\alpha>0$, and any $x \in \mathbb{R}$, write

$$
\left|f^{\alpha}(x)-f^{\alpha}(0)\right|=\alpha\left|\int_{0}^{x} f^{\alpha-1} f^{\prime} d x\right| \leq\left. C\left|\int_{0}^{x}\right| f V\right|^{\alpha-1}\left|f^{\prime}\right| \frac{1}{\rho_{a} V^{\alpha-1}} d \mu_{a} \mid .
$$

By the Hölder inequality, for any $p, q, r>1$ such that $1 / p+1 / q+1 / r=1$, then

$$
\left|\int f g h d \mu_{a}\right| \leq\|f\|_{p}\|g\|_{q}\|h\|_{r} .
$$

For $q=2, p=1 /(\alpha-1)$ and $r=2 /(3-2 \alpha)$ with $\alpha \in(1,3 / 2)$ this gives

$$
\left|f^{\alpha}(x)-f^{\alpha}(0)\right| \leq C U^{\alpha-1} \mathcal{E}_{\mu_{a}}(f, f)^{1 / 2} K^{\alpha}(x),
$$

where

$$
K(x)=\left|\int_{0}^{x} \frac{\rho_{a}(t)^{1-r}}{V(t)^{r(\alpha-1)}} d t\right|^{\frac{1}{r \alpha}}
$$

Then

$$
|f(0)| \leq|f(x)|+\left|f^{\alpha}(0)-f^{\alpha}(x)\right|^{1 / \alpha}
$$

for all $x$, since $\alpha \geq 1$, so

$$
|f(0)| \leq C\left[|f(x)|+U^{1-1 / \alpha} \mathcal{E}_{\mu_{a}}(f, f)^{1 /(2 \alpha)} K(x)\right],
$$

and then

$$
|f(0)| \int V d \mu_{a} \leq C\left(U+U^{1-1 / \alpha} \mathcal{E}_{\mu_{a}}(f, f)^{1 /(2 \alpha)} \int K V d \mu_{a}\right) .
$$

Let us prove that $\int K V d \mu_{a}$ is finite. By the definition (5.1) of $V$ and Corollaire 6.4.2 in [1] for instance, one has

$$
K(x)=\left|\int_{0}^{x} e^{\frac{T^{a}}{2}\left(\frac{3}{2} r-1\right)} T^{\beta r(\alpha-1)} d t\right|^{\frac{1}{r \alpha}} \leq C \exp \left(\frac{T^{a}(x)}{2}\right) T^{d}(x),
$$

with

$$
d=\beta\left(1-\frac{1}{\alpha}\right)-\frac{a-1}{r \alpha} .
$$

In fact the two quantities in (5.8) are equivalent when $|x|$ is large. 
Hence $K V \rho_{a} \leq C T^{d-\beta}$, so the integral $\int K V d \mu_{a}$ is convergent as soon as $d-\beta<-1$, that is,

$$
\alpha<1+\frac{1}{a}\left(\beta-\frac{3-a}{2}\right) .
$$

Hence, if $\beta>(3-a) / 2$, then any $1<\alpha<\min \left\{\frac{3}{2}, 1+\frac{1}{a}\left(\beta-\frac{3-a}{2}\right)\right\}$ satisfies all conditions, so that $\int K V d \mu_{a}<\infty$. Then

$$
\int|f-f(0)| V d \mu_{a} \leq C\left[U+U^{1-1 / \alpha} \mathcal{E}_{\mu_{a}}(f, f)^{1 /(2 \alpha)}\right]
$$

by (5.6) and (5.7). This proves Lemma 5.6 with $\theta=1 / \alpha$.

Remark 5.8. The argument is only based on the fact that the function

$$
K(x)=\left|\int_{0}^{x} \frac{\rho_{a}^{1-r}}{V^{r(\alpha-1)}} d t\right|^{\frac{1}{r \alpha}}
$$

satisfies

$$
\int K \rho_{a} V d x<\infty
$$

In particular, in the limiting case when $\beta=0$ and $V(x)=\rho_{a}^{-1 / 2}$, this amounts to

$$
\int_{0}^{\infty}\left[\int_{0}^{x} \rho_{a}(t)^{-\alpha /(3-2 \alpha)} d t\right]^{(3-2 \alpha) / 2 \alpha} \rho_{a}^{1 / 2}(x) d x<\infty,
$$

that is, $a>\frac{3}{3-2 \alpha}$ (see again Corollaire 6.4.2 in [1] for instance). In turn this holds for an $\alpha \in(1,3 / 2)$ if and only if $a>3$.

Remark 5.9. The two fundamental lemmas are based on the two estimates (5.2) and (5.8). These estimates are basic when proving that a probability measure on $\mathbb{R}$ satisfies a Poincaré or a logarithmic Sobolev inequalities, as explained in Section 6.4 of $[1]$.

Collecting lemmas 5.2 and 5.6, we get the following main result:

Theorem 5.10. On $\mathbb{R}$, let us consider the measure

$$
d \mu_{a}(x)=C_{a} \exp \left(-T^{a}\right) d x
$$

with $T(x)=\left(1+|x|^{2}\right)^{1 / 2}$, and the weight function

$$
V=\exp \left(\frac{T^{a}}{2}\right) T^{-\beta}
$$

with $a>1$ and $\beta \in \mathbb{R}$. Then there exist $C$ and $\lambda \in(0,1)$ such that

$$
\|f\|_{2}^{2} \leq C\left[\left(\int|f| V d \mu_{a}\right)^{2}+\left(\int|f| V d \mu_{a}\right)^{2(1-\lambda)} \mathcal{E}_{\mu_{a}}(f, f)^{\lambda}\right]
$$

for all functions $f$. 
Proof. The space of smooth functions with compact support is dense in the domain of $L$, so it is enough to consider the case when $f$ is smooth and compactly supported. Also, without loss of generality, we may assume that $f$ is non-negative. Here again $C$ will denote diverse constants depending on the parameters $a$ and $\beta$ and a parameter $\theta$ to be introduced later.

One has,

$$
\|f\|_{2}^{2} \leq\left(\int f d \mu_{a}\right)^{2}+\int|f-f(0)|^{2} d \mu_{a} .
$$

The weight $V$ is bounded from below by a positive constant, so

$$
\|f\|_{2}^{2} \leq C\left(\int f V d \mu_{a}\right)^{2}+\int|f-f(0)|^{2} d \mu_{a}
$$

Let now $U=\int f V d \mu_{a}$ and $U_{0}=\int|f-f(0)| V d \mu_{a}$ and assume $\beta>0$. By Lemma 5.2, applied to the function $f-f(0)$, one has

$$
\int|f-f(0)|^{2} d \mu_{a} \leq C \mathcal{E}_{\mu_{a}}(f, f)^{\gamma} U_{0}^{2(1-\gamma)}
$$

where

$$
\gamma=1-2 \frac{a-1}{3(a-1)+2 \beta}
$$

If, moreover, $\beta>(3-a) / 2$, by Lemma 5.6 there exists $\theta \in(0,1)$ such that

$$
U_{0} \leq C\left[U+U^{1-\theta} \mathcal{E}_{\mu_{a}}(f, f)^{\theta / 2}\right]
$$

so that

$$
\int|f-f(0)|^{2} d \mu_{a} \leq C \mathcal{E}_{\mu_{a}}(f, f)^{\gamma}\left[U^{2(1-\gamma)}+U^{2(1-\theta)(1-\gamma)} \mathcal{E}_{\mu_{a}}(f, f)^{\theta(1-\gamma)}\right]
$$

by (5.11). Hence, by (5.10),

$$
\begin{aligned}
\|f\|_{2}^{2} & \leq C U^{2}\left[1+\left(\frac{\mathcal{E}_{\mu_{a}}(f, f)}{U^{2}}\right)^{\gamma}+\left(\frac{\mathcal{E}_{\mu_{a}}(f, f)}{U^{2}}\right)^{\gamma+\theta(1-\gamma)}\right] \\
& \leq C\left(U^{2}+\mathcal{E}_{\mu_{a}}(f, f)^{\lambda} U^{2(1-\lambda)}\right)
\end{aligned}
$$

if $\lambda=\gamma+\theta(1-\gamma) \in(0,1)$. This concludes the argument for $\beta>\max \left(0, \frac{3-a}{2}\right)$.

Then, since $V$ is decreasing in $\beta$, then (5.9) holds for all real $\beta$.

Remark 5.11. We are restricted to $a>1$, since for $a=1$ then only $\lambda=1$ is admissible; this gives an inequality useless for our purpose, which is even weaker than the Poincaré inequality.

According to Lemma 5.6 and Remark 5.7 the larger $\beta$ is, the smaller the weight $V$ is, and the larger the exponent $\lambda$ of the Dirichlet form has to be in (5.9); on the contrary, the smaller $\beta$ is $(>3 / 2)$, the smaller we can take the exponent $\lambda$. 
We can now illustrate the abstract method of Section 3 by obtaining the following pointwise bounds on the Markov semigroup associated to $L$, which give new information on this semigroup for small time:

Corollary 5.12. Let $a>1$ and let $\left(P_{t}\right)_{t \geq 0}$ be the Markov generator on $\mathbb{R}$ with generator

$$
L f=f^{\prime \prime}-a T^{a-1} T^{\prime} f^{\prime},
$$

and reversible measure $d \mu_{a}(x)=\rho_{a}(x) d x=C_{a} \exp \left(-\left(1+|x|^{2}\right)^{a / 2}\right) d x$.

Then for all real $\beta$ there exists $\delta>0$ and a constant $C$ such that, for all $t, P_{t}$ has a density $p_{t}$ with respect to the measure $\mu_{a}$, which satisfies

$$
p_{t}(x, y) \leq \frac{C e^{C t}}{t^{\delta}} \frac{\rho_{a}^{-1 / 2}(x) \rho_{a}^{-1 / 2}(y)}{\left(1+|x|^{2}\right)^{\beta / 2}\left(1+|y|^{2}\right)^{\beta / 2}}
$$

for almost every $x, y \in \mathbb{R}$.

Moreover, the spectrum of $-L$ is discrete and its eigenvalues $\left(\lambda_{n}\right)_{n \in \mathbb{N}}$ satisfy the inequality

$$
\sum_{n} e^{-\lambda_{n} t} \leq \frac{C e^{C t}}{t^{\delta}}
$$

for all $t>0$.

Proof. Letting $C$ and $\lambda \in(0,1)$ be defined as in Theorem 5.10, by the inequality (5.9) the Dirichlet form $\mathcal{E}_{\mu_{a}}$ satisfies a weighted Nash inequality with weight $V=\exp \left(T^{a} / 2\right) T^{-\beta}$ and rate function

$$
\phi(x)=C^{-1 / \lambda}(x-C)^{1 / \lambda}
$$

on $(C,+\infty)$. Moreover the weight $V$ is a Lyapunov function with constant $c>0$ by Lemma 5.1 , it is in $\mathcal{L}^{2}\left(\mu_{a}\right)$ if $\beta>1 / 2$ and hypothesis (3.4) of Theorem 3.5 holds since $\lambda<1$. Hence, by Corollary 3.7 and for diverse constants $C=C(a, \beta, \lambda)$, for all $t>0$ the operator $P_{2 t}$ has a density $p_{2 t}$ with respect to $\mu_{a}$, which satisfies

$$
\begin{aligned}
p_{2 t}(x, y) & \leq C\left(1+t^{\frac{-2 \lambda}{1-\lambda}}\right) e^{2 c t} \frac{\rho_{a}^{-1 / 2}(x) \rho_{a}^{-1 / 2}(y)}{\left(1+|x|^{2}\right)^{\beta / 2}\left(1+|y|^{2}\right)^{\beta / 2}} \\
& \leq C t^{\frac{-2 \lambda}{1-\lambda}} e^{2 c t} \frac{\rho_{a}^{-1 / 2}(x) \rho_{a}^{-1 / 2}(y)}{\left(1+|x|^{2}\right)^{\beta / 2}\left(1+|y|^{2}\right)^{\beta / 2}} .
\end{aligned}
$$

This proves the first statement for $\beta>1 / 2$, with $\delta=2 \lambda /(1-\lambda)>0$, and then for any $\beta$.

The second statement on the trace of $P_{t}$ is obtained by taking any $\beta>1 / 2$ in the upper bound on $p_{t}(x, x)$ and integrating.

For $\beta>1 / 2$, the non-uniform bound implies that $P_{t}$ is Hilbert-Schmidt but we do not recover the Orlicz hypercontractivity result of [7]. This is not surprising since in fact no bound such as $K(t) V(x) V(y)$ can imply hypercontractivity or more generally Orlicz hypercontractivity. 
Remark 5.13. The same method, with $V=1$, leads to a (non-weighted) Nash inequality for $\mu_{a}$ with $a>1$ and with rate function

$$
\phi(x)=C x(\log x)^{2(1-1 / a)}
$$

on an interval $(M, \infty)$. By Theorem 3.5 this implies that the semigroup is ultracontractive as soon as $1 / \phi$ is integrable at infinity, that is, for $a>2$, hence recovering a partial result of [18].

Remark 5.14. Observe in Corollary 5.12 that there is no optimal $\beta$, that is, no optimal bound on $p_{t}(x, x)$ of the form $C(t) \rho(x)^{-1 / 2} T(x)^{-\beta}$. So one could look for an optimal bound on $p_{t}(x, x)$ such as $C(t) \rho^{-\lambda}(x)$ for a $\lambda \in(0,1 / 2)$. This is not the case in the Gaussian case when $a=2$. In this case the optimal bound is $C(t) \exp \left(|x|^{2} /\left(1+e^{2 t}\right)\right)$, hence of the form $C(t) \rho(x)^{-\lambda(t)}$ with $\lambda(t)<1 / 2$; it is even an equality, see (3.1).

Also for $1<a<2$ it seems that $p_{t}(x, x)$ cannot be bounded by $C(t) \rho^{-\lambda}(x)$ for $\lambda<1 / 2$. Indeed, for the weight $V=\exp \left(\lambda T^{a}\right)$ with $\lambda<1 / 2$, our method leads to a weighted Nash inequality with rate function

$$
\phi(x)=C(a, \lambda) x(\log x)^{2(1-1 / a)}
$$

on an interval $(M, \infty)$, where $\lambda$ appears only in the value of the constant $C(a, \lambda)$. Apart from the values of the constants, this is not better than the inequality obtained in Remark 5.13 with $V=1$, and again this is not enough to obtain any bound on the density $p_{t}(x, y)$, by the lack of integrability of $1 / \phi$. Now we do not know whether a bound such as $C(t) \rho(x)^{-\lambda(t)}$ with $\lambda(t)<1 / 2$ could be optimal, but we strongly doubt it.

Again from this point of view the Gaussian case appears as a particular case, being a critical case as regards the two points of view of ultracontractivity and non-uniform bounds. In this case, and in this case only, one may do better, and Gaussian Nash inequalities are under study in a work in progress.

Acknowledgements. We would like to thank F.-Y. Wang for pointing out his work [28] to us, and the referee for pointing out reference [31].

\section{References}

[1] Ané, C., Blachère, S., Chafaï, D., Fougères, P., Gentil, I., Malrieu, F., Roberto, C. And Scheffer, G.: Sur les inégalités de Sobolev logarithmiques. Panoramas et Synthèses, 10. Société Mathématique de France, Paris, 2000.

[2] BAKRy, D.: L'hypercontractivité et son utilisation en théorie des semigroupes. In Lectures on probability theory (Saint-Flour, 1992), 1-14. Lecture Notes in Math. 1581. Springer, Berlin, 1994.

[3] Bakry, D., Barthe, F., Cattiaux, P. and Gulllin, A.: A simple proof of the Poincaré inequality for a large class of probability measures including the log-concave case. Electron. Commun. Probab. 13 (2008), 60-66. 
[4] Bakry, D, Bolley, F. And Gentil, I.: Dimension dependent hypercontractivity of gaussian kernels. To appear in Probab. Theory and Related Fields (2012).

[5] Bakry, D., Cattiaux, P. And Guillin, A. : Rate of convergence for ergodic continuous Markov processes: Lyapunov versus Poincaré. J. Funct. Anal. 254 (2008), no. 3, 727-759.

[6] Bakry, D., Coulhon, T., Ledoux, M. and Saloff-Coste, L.: Sobolev inequalities in disguise. Indiana Univ. Math. J. 44 (1995), no. 4, 1033-1074.

[7] Barthe, F., Cattiaux, P. And Roberto, C.: Interpolated inequalities between exponential and Gaussian, Orlicz hypercontractivity and isoperimetry. Rev. Mat. Iberoam. 22 (2006), no. 3, 993-1067.

[8] Barthe, F., Cattiaux, P. and Roberto, C.: Isoperimetry between exponential and Gaussian. Electron. J. Probab. 12 (2007), no. 44, 1212-1237.

[9] Bendikov, A., Coulhon, T. And Saloff-Coste, L.: Ultracontractivity and embedding into $L^{\infty}$. Math. Ann. 337 (2007), no. 4, 817-853.

[10] Boвkov, S. G.: Isoperimetric and analytic inequalities for log-concave probability measures. Ann. Probab. 27 (1999), no. 4, 1903-1921.

[11] Boвkov, S. G. And Götze, F.: Exponential integrability and transportation cost related to logarithmic Sobolev inequalities. J. Funct. Anal. 193 (1999), no. 1, 1-28.

[12] Carlen, E. A., Kusuoka, S. and Stroock, D. W.: Upper bounds for symmetric Markov transition functions. Ann. Inst. H. Poincaré Probab. Statist. 23 (1987), no. 2, suppl., 245-287.

[13] Carlen, E. A. And Loss, M.: Sharp constant in Nash's inequality. Internat. Math. Res. Notices 1993, no. 7, 213-215.

[14] Coulhon, T.: Ultracontractivity and Nash type inequalities. J. Funct. Anal. 141 (1996), no. 2, 510-539.

[15] Davies, E. B.: Heat kernels and spectral theory. Cambridge Tracts in Mathematics, 92. Cambridge University Press, Cambridge, 1990.

[16] Gentil, I., Guillin, A. And Miclo, L.: Modified logarithmic Sobolev inequalities in null curvature. Rev. Mat. Iberoam. 23 (2007), no. 1, 235-258.

[17] Gross, L.: Logarithmic Sobolev inequalities. Amer. J. Math. 97 (1975), no. 4, 1061-1083.

[18] Kavian, O., Kerkyacharian, G. And Roynette, B.: Quelques remarques sur l'ultracontractivité. J. Funct. Anal. 111 (1993), no. 1, 155-196.

[19] Kolmogorov, A., Fomine, S. And Tihomirov, V. M.: Eléments de la théorie des fonctions et de l'analyse fonctionnelle. Éditions Mir, Moscow, 1974.

[20] Latąa, R. And Oleszkiewicz, K.: Between Sobolev and Poincaré. In Geometric aspects of functional analysis, 147-168. Lecture Notes in Math., 1745. Springer, Berlin, 2000.

[21] Ledoux, M.: The geometry of Markov diffusion generators. Ann. Fac. Sci. Toulouse Math. (6) 9 (2000), no. 2, 305-366.

[22] Maheux, P.: Nash-type inequalities and decay of semigroups of operators. Preprint (2010).

[23] Muckenhoupt, B.: Hardy's inequality with weights. Studia Math. 44 (1972), $31-18$.

[24] NAsh, J.: Continuity of solutions of parabolic and elliptic equations. Amer. J. Math. 80 (1958), 931-954. 
[25] Saloff-Coste, L.: Sobolev inequalities in familiar and unfamiliar settings. In Sobolev spaces in mathematics. I, 299-343. Int. Math. Ser., 8. Springer, New York, 2009 .

[26] Varopoulos, N. Th., Saloff-Coste, L. and Coulhon, T.: Analysis and geometry on groups. Cambridge Tracts in Mathematics, 100. Cambridge University Press, Cambridge, 1992.

[27] Wang, F.-Y.: Functional inequalities for empty essential spectrum. J. Funct. Anal. 170 (2000), no. 1, 219-245.

[28] WANG, F.-Y.: Functional inequalities and spectrum estimates: the infinite measure case. J. Funct. Anal. 194 (2002), no. 2, 288-310.

[29] Wang, F.-Y.: Functional inequalities, Markov processes and spectral theory. Science Press, Beijing, 2004.

[30] Wang, F.-Y.: A generalization of Poincaré and log-Sobolev inequalities. Potential Anal. 22 (2005), no. 1, 1-15.

[31] WAng, F.-Y.: Intrinsic ultracontractivity on Riemannian manifolds with infinite volume measures. Sci. China Math. 53 (2010), no. 4, 895-904.

[32] Yosida, K.: Functional analysis. Classics in Mathematics. Springer-Verlag, Berlin, 1995.

Received June 24, 2010; revised June 1, 2011.

Dominique BAKRY: Institut de Mathématiques de Toulouse and Institut Universitaire de France, UMR CNRS 5219, Université de Toulouse, Route de Narbonne, 31062 Toulouse, France.

E-mail: bakry@math.univ-toulouse.fr

François Bolley: Ceremade, UMR CNRS 7534, Université Paris-Dauphine, Place du Maréchal De Lattre De Tassigny, 75016 Paris, France.

E-mail: bolley@ceremade.dauphine.fr

Ivan Gentil: Institut Camille Jordan, UMR CNRS 5208, Université Claude Bernard Lyon 1, 43 boulevard du 11 novembre 1918, 69622 Villeurbanne, France.

E-mail: gentil@math.univ-lyon1.fr

Patrick Maheux: Mapmo, UMR CNRS 6628, Université d'Orléans, Bâtiment de mathématiques, Route de Chartres, 45067 Orléans, France.

E-mail: patrick.maheux@univ-orleans.fr

This research was supported in part by the ANR project EVOL. The third author thanks the members of UMPA at the Ecole Normale Supérieure de Lyon for their kind hospitality. 